\title{
CONCEPCIONES PEDAGÓGICAS Y DIDÁCTICAS DE LAS POLÍTICAS, PLANES, PROGRAMAS Y PROYECTOS DE ACTIVIDAD FÍSICA A NIVEL LOCAL Y NACIONAL
}

\author{
TEACHING AND DIDACTIC PRINCIPLES BY POLICY, PLANNING, PROGRAMS AND PROJECTS \\ OF PHYSICAL ACTIVITY AT DOMESTIC AND NATIONAL LEVEL
}

\author{
Alberto Calderón García ${ }^{1}$ \\ Olegario de Jesús Puentes Torres ${ }^{2}$ \\ Víctor Hugo Durán Camelo 3 \\ Ramiro Andrés AlzateLubo ${ }^{4}$ \\ Gloria Viviana Barinas Prieto ${ }^{5}$
}

Resumen

Esta investigación, desarrollada en 2008 por el grupo investigación Gestión y Pedagogía de la Actividad Física -GPAF-, de la Universidad Pedagógica Nacional, indagó acerca de las concepciones pedagógicas y didácticas que atraviesan las políticas, planes, programas y proyectos de actividad física en contextos nacionales, a partir de un abordaje multidimensional y multisectorial. Teniendo en cuenta los objetivos y características del estudio planteado, se utilizaron los siguientes métodos: a) análisis del discurso pedagógico,b) análisis documental del marco legislativo, político y administrativo, c) análisis comparativo de las diferentes posturas conceptuales actuales de actividad física, d) análisis descriptivo de la categorización y tabulación de los instrumentos y e) análisis documental y comparativo de los programas exitosos de actividad física en la Guajira, Bogotá y Antioquia. Con los resultados de la intervención metodológica se detectó poca importancia de los constructos de pedagogía y didáctica en la política social, como una forma de intervención del Estado en la sociedad civil para fomentar servicios y brindar programas de actividad física, y lograr un cambio en el orden social donde se evidencie la reducción de factores de riesgo en salud. Además, los hallazgos indicaron que en la actualidad las políticas, planes, programas y proyectos desarrollados por el Estado colombiano, se construyen y desarrollan desde una perspectiva más administrativo-asistencialista que limita en parte las intenciones educativas, las estrategias pedagógicas y didácticas relacionadas con la práctica social de la actividad física.

Palabras clave: Pedagogía, didáctica, concepciones pedagógicas, actividad física, políticas, planes, programas, pro yectos.

1 Investigador principal. Ph.D. en Administración y Formación Profesional, Educación Física y Deporte, y M.Sc. en Salud y Educación Física. Docente Universidad Pedagógica Nacional. Correo electrónico: acalderon@pedagogica.edu.co

2 Coinvestigador. Especialista en Administración de la Educación Física, Deporte y Recreación .Docente Universidad Pedagógica Nacional. Correo electrónico: opt276@gmail.com

3 Coinvestigador. Estudiante de Doctorado Interinstitucional de Educación. Docente Universidad Pedagógica Nacional. Correo electrónico: guajiro78@gmail.com

4 Coinvestigador. Estudiante de Maestría en Educación Universidad Pedagógica Nacional. Correo electrónico: ramironcho@yahoo.com

5 Licenciada en Biología. Candidata a Magíster en Educación de la Universidad Pedagógica Nacional. Becaria del programa “Jóvenes investigadores e innovadores" de Colciencias. 


\section{Abstract}

This research conducted in 2008 by the group "Management and Pedagogy of Physical Activity" (GPAF) at UPN explored from a multidimensional and multiregional approach some teaching and didactic principles based on policy, planning, programs and projects of physical activity at national level. Taking into account goals and features proposed by the study, the following methods were used: a) a pedagogic discourse analysis; b) a document analysis of a legal, political and administrative framework; c) a comparative analysis of current several conceptual perspectives of physical activity; d) a descriptive analysis of tool categorization and classification; and e) a comparative-documentary analysis of successful programs of physical activity in Guajira, Bogotá and Antioquia. Results show that current policies, plans, programs and projects developed by Colombian state are built and developed from an administrative-welfare focus, rather than educational purpose, strategies related to education and teaching social practice of the physical activity .

Keywords: concepts, pedagogy, didactics, education, physical activity, policies, plans, programs, projects.

Fecha de recepción: 18 de mayo de 2012

Fecha de aprobación: 19 de septiembre de 2012

\section{Introducción}

La creciente importancia que tiene la actividad física y la salud en el contexto de la globalización, permite trabajar en diversos campos desde los puntos de vista científico, político, mediático y socioeconómico que han intervenido en su abordaje para mejorar las condiciones de prevención de enfermedades crónicas no transmisibles y la promoción de la salud. Existen, en este sentido, diversos estudios sociales que han dirigido su mirada a escudriñar acerca de los beneficios de la actividad física para los adultos, niños y jóvenes en edad escolar, familias, comunidades y entidades de salud, tomando en cuenta su nivel socioeconómico, motivación y cesación en su práctica.

Este estudio se basa en el análisis y caracterización de las concepciones desde la óptica pedagógica sobre las políticas, planes, programas y proyectos de actividad física, aprovechando teorías y constructos educativos y pedagógicos y acciones didácticas relacionadas con los ámbitos comunitario, laboral, escolar y de salud. De estos se escogió para el presente trabajo el comunitario, a partir del cual surgió el análisis de los conceptos multisectorial, interinstitucional e interdisciplinario; se pretendió, con este enfoque pedagógico, interrogar acerca de la pertinencia y concordancia de los planes, programas y proyectos con las necesidades que presentan los diversos actores sociales dentro de este proceso, con respecto a la actividad física y su relación con la salud en los contextos locales y nacionales.

\section{Marco de referencia}

1. Pedagogía. La pedagogía se conceptualiza desde diferentes ángulos. como se observa a continuación:

- La pedagogía como hacer reflexivo ubica al docente como centro del proceso de enseñanzaaprendizaje, atribuyéndole la responsabilidad de reflexionar sobre la educación formal y la educación para el trabajo y el desarrollo humano, de acuerdo con la Ley 1064/06. Según Lucio (1996), "saber educar" se convierte en un "saber sobre la educación", sobre sus cómos, sus porqués y sus hacia dónde".

- La pedagogía como discurso enfatiza el aprendizaje como centro, que implica una estructura de "co-definiciones" sobre lo epistemológico, didáctico, las concepciones del profesor y su rol, la enseñanza, el estudiante y su papel en el apren- 
Concepciones pedagógicas y didácticas de las políticas, planes, programas y proyectos de acti-

vidad física a nivel local y nacional. Alberto Calderón García, Olegario de Jesús Puentes Torres,

Víctor Hugo Durán Camelo, Ramiro Andrés Alzate Lubo y Gloria Viviana Barinas Prieto

dizaje. La pedagogía es discurso en la medida que tematiza sobre el aprendizaje en asocio con las co-definiciones mencionadas, según Gallego y Pérez, (1988). Por su parte, Zuluaga (1988) señala al respecto que la pedagogía es "un discurso acerca de la enseñanza y, a la vez, como una práctica cuyo campo de aplicación es el discurso".

- A la pedagogía como saber disciplinar, Zuluaga (1988) la establece como "disciplina que conceptualiza, aplica y experimenta los conocimientos referentes a la enseñanza de los saberes específicos en las diferentes culturas. Se refiere tanto a los procesos de enseñanza propia de la exposición de las ciencias, como al ejercicio del conocimiento en la interioridad de la cultura".

- La pedagogía como ciencia es interpretada como ciencia social, que tiene como objeto de estudio el hecho educativo. Camacho (2003), le atribuye la responsabilidad de liderar la formación del hombre para que responda a las demandas de la sociedad a la que pertenece. Así mismo, Lucio (1990) la presenta como el "proceso por el cual la sociedad facilita de una manera intencional o implícita el crecimiento de sus miembros", pues "la educación es ante todo una práctica social, que lleva implícita una visión de hombre".

- Corrientes pedagógicas. En el devenir histórico y teórico de la educación se han inscrito dos tendencias: la pedagogía y la didáctica, que parecen opuestas y determinan el rol tanto del sujeto enseñante, como el del sujeto del aprendizaje. Las relaciones que se establecen a partir de estas posturas, colaboran también a la aproximación del conocimiento, la estructura de los contenidos y el método de transmisión de esos contenidos.

- La pedagogía crítica articula las necesidades educativas de la población y el requerimiento de generar procesos de constitución del sujeto para integrarse políticamente en las dinámicas sociales.

En esta investigación se asumió el concepto de pedagogía crítica para problematizar el objeto de estudio sobre el aprendizaje, con los diferentes factores que la componen como contextos, sujetos, prácticas y saberes.
Desde lo anterior, se rastreó lo pedagógico teniendo en cuenta: 1. la presencia de una visión de hombre; 2 . concepciones sobre conocimiento, educación, hombre y sociedad, realidad sociohistórica, modelos pedagógicos, aprendizaje y procesos de aprendizaje; 3 . sentido educativo de la propuesta; 4 . impacto social desde lo formativo.

2. Didáctica. Presenta una variedad de concepciones, bien sea que se mire de manera general o particular o en relación con su objeto de estudio, desde donde se analiza como disciplina, como saber pedagógico, como tecnología educativa y como modelo didáctico y socio-crítico:

- La didáctica como disciplina tematiza el proceso de instrucción y orienta sus métodos, estrategias, eficiencia, etc. (Lucio, 1996). En otras palabras, la didáctica es la disciplina cuyo objeto de estudio se centra en la enseñanza. Según este autor, la didáctica debe estar orientada por un pensamiento pedagógico, en razón a que la enseñanza es "un momento específico de la práctica educativa".

- La didáctica como saber hace parte de una estructura organizacional en que la educación es la categoría mayor, la pedagogía es la intermedia y, finalmente, la didáctica es la base como saber pedagógico "más o menos delimitado", según Vasco (1985).

- La didáctica como tecnología educativa es la encargada de la organización de contenidos y procedimientos de enseñanza que se suceden en situaciones de aprendizaje, eventualmente jerarquizadas, de la cual dependen objetivos y estrategias pedagógicas adoptadas en la disciplina que se desarrolla. (Camacho, 2003).

- Modelos didácticos. Desde la educación física se plantean modelos didácticos que responden, según su estructura, a dos tendencias o modelos: directivos y no directivos. Camacho (2003) los divide en estilo tradicional o decadente, que no posibilita la participación del estudiante y está centrado en el maestro, y el tecnocrático o dominante, centrado en el estudiante.

- Flórez (1994) caracteriza las tendencias didácticas desde la postura del docente en contraposición a la del estudiante, en virtud al rol que cada uno asume como agente del aprendizaje ante el fundamento epistemológico, el modelo normativo y las relaciones de poder que se establecen. 
- Didáctica socio-crítica. La postura didáctica tiene en cuenta el contexto y está relacionada con las dinámicas sociales, condiciones políticas y socioeconómicas actuales.

En este sentido, se aborda la didáctica no como un conjunto de técnicas, sino como una disciplina que está en proceso de construcción (Martínez, Guzmán y Calderón, 2005). En esa línea de pensamiento y para hacer una distinción fundamental en el presente estudio, la "didáctica" como disciplina se encarga de problematizar el objeto de estudio sobre la enseñanza, con los diferentes factores que la componen: estructura curricular, métodos, contenidos, estrategias de enseñanza y relaciones.

Concepciones pedagógicas. En este estudio, el término "concepciones" se distingue conceptual y metodológicamente de otras teorías como son las representaciones sociales, sin negar que en cierta forma las concepciones son una forma de representación.

Las "concepciones" son construcciones que se generan a partir de los saberes previos y esquemas conceptuales de los sujetos. Esta definición advierte, en primer lugar, que frente a los conceptos todo ser humano presenta presupuestos que le llevan a intuir una forma de definir el concepto mismo y su relación con la realidad, lo cual comparte con las representaciones sociales, y en segundo lugar, las concepciones pedagógicas necesitan de fundamentos epistémicos que la sustenten y requiere de una postura pedagógica y ética de la intencionalidad y aplicabilidad en lo real.

Por último, se puede decir que las "concepciones" hacen parte de la estructura conceptual de un saber educativo o pedagógico, pues los actores que fomentan la actividad física desde las políticas, programas y proyectos tienen "o deberían tener" una postura frente a la misma, producto de su formación o experiencia, lo cual diferenciaría las concepciones de las representaciones sociales, que según Moscovici (1979) son una modalidad particular del conocimiento espontáneo cuya función es la elaboración de los comportamientos y la comunicación entre los individuos.

3. Políticas. Se entienden como designación de los propósitos y programas de las autoridades públicas.

Desde la óptica de las políticas públicas, estas corresponden a las "acciones decididas llevadas a cabo y autorizadas por el estado" (Roth, 2006). Para dicho efecto, debe entenderse por lo público lo que abarca la dimensión de la actividad humana que requiere de una intervención gubernamental o social, o por lo menos una acción en común.

Al proyectar una política pública hacia la actividad física y sus beneficios, se podría entender como un programa de acción que representa la concreción de una serie de decisiones, promulgadas a través de textos jurídicos y administrativos que sirven para satisfacer aquellos que tengan que ver con el bienestar integral de los ciudadanos.

Las políticas públicas concretan lo que un Estado o sociedad considera como de interés público o de interés general, y son el enlace entre el Estado, la sociedad y el ciudadano. Debe aclararse que "la simple expedición de una norma no constituye por sí sola una política pública, y menos una garantía de efectividad, pero sí señala lo que se considera de interés público aquí y ahora" (Roth, 2006).

Para intervenir en la sociedad a fin de modificar los comportamientos de los actores sociales, razón de ser de las políticas públicas, el Estado como estructura específica de poder tiene instrumentos de intervención diversificados. La efectividad de los instrumentos utilizados, expresados casi siempre bajo una forma jurídica, pueden ser una ayuda importante; pero una política pública no se limita a instrumentos jurídicos, pues estos representan solo una formulación de la solución que se requiere; su impacto concreto se obtendrá mediante la movilización coordinada de diferentes recursos.

Planes. Su propósito debe ser el logro de los fines marcados. Estos, a su vez, deben estructurarse en unidades más concretas y específicas que persiguen la satisfacción de objetivos: para hablar de programas de actuación cada objetivo se subdivide en las unidades de ejecución, para llegar a los proyectos, último eslabón teórico que se enlaza directamente con la puesta en práctica de todo el proceso. Un proceso que no llegue a ejecutarse puede admitir otras denominaciones, para no definirse como un plan. Es importante tener en cuenta que un plan no culmina con la elaboración teórica del proceso, ni siquiera con la puesta en práctica: finaliza con la evaluación y la toma de decisiones oportunas.

Programas. Permitirán que antes de emprender cualquier acción, se establezcan la cronología y la priori- 
Concepciones pedagógicas y didácticas de las políticas, planes, programas y proyectos de acti-

vidad física a nivel local y nacional. Alberto Calderón García, Olegario de Jesús Puentes Torres,

Víctor Hugo Durán Camelo, Ramiro Andrés Alzate Lubo y Gloria Viviana Barinas Prieto

dad de cada una de las etapas que conduzcan al resultado final. Al programar se estará, de hecho, contando con un medio para reconocer diversas alternativas $\mathrm{y}$, en consecuencia, buscar soluciones para decidir de forma más rápida y económica cómo alcanzar el objetivo concretado.

Un programa presenta una serie de etapas que se deben ir alcanzando para lograr los objetivos, en donde cada una exigirá una determinada cantidad de trabajo y de esfuerzo tanto físico como mental. El programa se puede considerar también como un plan que incluye el uso futuro de diversos recursos y que establece una secuencia de acciones para lograr un determinado objetivo.

Es importante notar que el trabajo intersectorial a nivel nacional enfocado hacia los programas de actividad física está en una etapa naciente, motivo por el cual se requiere de una mejor estructura teórica que servirá como soporte para las futuras sistematizaciones y las investigaciones que se hagan en este campo.

Para lograr que la promoción de la actividad física sea una prioridad dentro de los respectivos planes de desarrollo que se formulen, estos deben ser entendidos como un proceso que debe ser asumido de manera responsable y democrática por la respectiva autoridad, de tal forma que se convierta en un instrumento de gestión para la administración a través del cual se puedan conocer, definir y priorizar los problemas, mediado por la acción entre el respectivo gobierno y la comunidad.

Tabla 1. Corrientes epistemológicas y conceptuales de la actividad física. (Calderón y Alzate, 2010)

\begin{tabular}{|c|c|c|c|c|}
\hline \multicolumn{5}{|c|}{ CONCEPTOS DE ACTIVIDAD FÍSICA } \\
\hline $\begin{array}{l}\text { Corrientes } \\
\text { ceptuales }\end{array}$ & con- & \multirow{2}{*}{$\begin{array}{l}\text { Concepto de A.F. } \\
\text { (Pedraz) }\end{array}$} & Concepto de A.F. & \multirow{2}{*}{$\begin{array}{l}\text { Concepto de A.F } \\
\text { desde el enfoque } \\
\text { psicológico am- } \\
\text { biental y comu- } \\
\text { nitario }\end{array}$} \\
\hline $\begin{array}{l}\text { Aspectos } \\
\text { rales }\end{array}$ & gene- & & $\begin{array}{l}\text { Center for Disease } \\
\text { Control and Pre- } \\
\text { vention (CDC) }\end{array}$ & \\
\hline \multirow[t]{2}{*}{$\begin{array}{l}\text { Definición } \\
\text { ceptual }\end{array}$} & \multirow[t]{2}{*}{ con- } & $\begin{array}{l}\text { Son todas aquellas } \\
\text { prácticas corpo- } \\
\text { rales que están } \\
\text { mediadas por } \\
\text { el movimiento: } \\
\text { ejercicio, deporte, } \\
\text { práctica motriz, } \\
\text { etc. }\end{array}$ & $\begin{array}{l}\text { Actividades mo- } \\
\text { trices cotidianas, } \\
\text { como caminar en } \\
\text { forma regular y } \\
\text { rítmica, jardinería, } \\
\text { tareas domésticas } \\
\text { pesadas y baile. }\end{array}$ & \multirow{2}{*}{$\begin{array}{l}\text { Interacción diná- } \\
\text { mica de factores } \\
\text { intrapersonales y } \\
\text { ambientales que } \\
\text { se regulan por } \\
\text { procesos cogni- } \\
\text { tivos, afectivos y } \\
\text { sociales. }\end{array}$} \\
\hline & & $\begin{array}{l}\text { Cualidad del ser } \\
\text { humano más allá } \\
\text { de lo puramente } \\
\text { biológico y fun- } \\
\text { cional. }\end{array}$ & $\begin{array}{l}\text { Movimientos cor- } \\
\text { porales planifica- } \\
\text { dos, estructurados } \\
\text { y repetitivos, para } \\
\text { mejorar y/o man- } \\
\text { tener la salud física. }\end{array}$ & \\
\hline
\end{tabular}

\begin{tabular}{|c|c|c|c|}
\hline Objetivo & $\begin{array}{l}\text { Desarrolla sujeto } \\
\text { integral. }\end{array}$ & $\begin{array}{l}\text { Incidir en el } \\
\text { estado de salud. Sus } \\
\text { fundamentos son: } \\
\text { la prevención de en- } \\
\text { fermedades cróni- } \\
\text { cas y la promoción } \\
\text { de la salud. }\end{array}$ & $\begin{array}{l}\text { Desarrolla sujeto } \\
\text { integral. }\end{array}$ \\
\hline $\begin{array}{l}\text { Objeto de } \\
\text { estudio }\end{array}$ & $\begin{array}{l}\text { El ser humano en } \\
\text { movimiento y la } \\
\text { incidencia de este } \\
\text { en el entorno, como } \\
\text { parte esencial de su } \\
\text { proceso educativo. }\end{array}$ & $\begin{array}{l}\text { El cuerpo funcional } \\
\text { del individuo en } \\
\text { búsqueda de su } \\
\text { bienestar. }\end{array}$ & $\begin{array}{l}\text { Procesos cogni- } \\
\text { tivos, afectivos } \\
\text { y sociales para } \\
\text { la escogencia } \\
\text { individual de ser } \\
\text { o no activo. }\end{array}$ \\
\hline Metodología & $\begin{array}{l}\text { Reflexión y } \\
\text { comprensión } \\
\text { de diferentes } \\
\text { posibilidades de } \\
\text { movimiento para } \\
\text { adaptarse al mun- } \\
\text { do en su proceso } \\
\text { de crecimiento y } \\
\text { desarrollo. }\end{array}$ & $\begin{array}{l}\text { A través de } \\
\text { movimientos } \\
\text { que implican la } \\
\text { participación de } \\
\text { órganos y sistemas } \\
\text { especializados, con } \\
\text { miras a la obtención } \\
\text { de beneficios sobre } \\
\text { el estado de salud } \\
\text { del individuo. }\end{array}$ & $\begin{array}{l}\text { Relaciona el gra- } \\
\text { do de escogencia } \\
\text { del individuo y } \\
\text { algunos factores } \\
\text { internos y } \\
\text { externos, que } \\
\text { pueden influir en } \\
\text { los procesos de } \\
\text { vinculación de } \\
\text { los usuarios. }\end{array}$ \\
\hline $\begin{array}{l}\text { Ciencias o } \\
\text { disciplinas que } \\
\text { intervienen }\end{array}$ & $\begin{array}{l}\text { Pedagogía, didác- } \\
\text { tica, psicología, } \\
\text { sociología, antro- } \\
\text { pología, historia, } \\
\text { biología, fisiología, } \\
\text { biomecánica, entre } \\
\text { otras. }\end{array}$ & $\begin{array}{l}\text { Ciencias médicas, } \\
\text { biología, fisiología, } \\
\text { kinesiología, biome- } \\
\text { cánica, antropo- } \\
\text { metría, ergonomía, } \\
\text { fisioterapia, entre } \\
\text { otras. }\end{array}$ & $\begin{array}{l}\text { Sociología, ecolo- } \\
\text { gía y desarrollo } \\
\text { urbanístico, } \\
\text { entre otras. }\end{array}$ \\
\hline $\begin{array}{l}\text { Tipos de } \\
\text { sujetos que } \\
\text { participan }\end{array}$ & $\begin{array}{l}\text { Todas las personas } \\
\text { que hacen parte } \\
\text { en su proceso } \\
\text { evolutivo de desa- } \\
\text { rrollo filogenético y } \\
\text { ontogenético. }\end{array}$ & $\begin{array}{l}\text { Personas y/o } \\
\text { grupos específicos } \\
\text { estableciendo su } \\
\text { ámbito y necesi- } \\
\text { dades. }\end{array}$ & $\begin{array}{l}\text { Personas y/o } \\
\text { grupos específi- } \\
\text { cos establecien- } \\
\text { do su ámbito y } \\
\text { necesidades. }\end{array}$ \\
\hline $\begin{array}{l}\text { ¿Quién eje- } \\
\text { cuta? }\end{array}$ & $\begin{array}{l}\text { El líder o el indi- } \\
\text { viduo. }\end{array}$ & $\begin{array}{l}\text { El individuo, } \\
\text { previo diagnóstico } \\
\text { y prescripción espe- } \\
\text { cializada. }\end{array}$ & $\begin{array}{l}\text { Actores indivi- } \\
\text { duales o sociales. }\end{array}$ \\
\hline \multirow[t]{2}{*}{ Infraestructura } & \multirow{2}{*}{$\begin{array}{l}\text { Requiere un } \\
\text { espacio al aire } \\
\text { libre o cerrado } \\
\text { con equipos y } \\
\text { material didáctico } \\
\text { o deportivo. }\end{array}$} & $\begin{array}{l}\text { Requiere un espacio } \\
\text { al aire libre o cerra- } \\
\text { do con equipos y } \\
\text { material de poco o } \\
\text { ningún costo. }\end{array}$ & $\begin{array}{l}\text { Requiere un } \\
\text { espacio al aire } \\
\text { libre o cerrado } \\
\text { con equipos y } \\
\text { material de poco } \\
\text { o ningún costo. }\end{array}$ \\
\hline & & $\begin{array}{l}\text { Pueden utilizarse } \\
\text { equipos biomédicos } \\
\text { especializados. }\end{array}$ & $\begin{array}{l}\text { Pueden utilizarse } \\
\text { equipos biomé- } \\
\text { dicos especiali- } \\
\text { zados. }\end{array}$ \\
\hline Ámbitos & $\begin{array}{l}\text { Escolar } \\
\text { Comunitario }\end{array}$ & $\begin{array}{l}\text { Salud } \\
\text { Escolar } \\
\text { Laboral } \\
\text { Comunitario }\end{array}$ & $\begin{array}{l}\text { Social } \\
\text { Comunitario }\end{array}$ \\
\hline
\end{tabular}

Proyectos. Se pueden definir como el propósito de emprender una actividad o conjunto de actividades para conseguir un objetivo común, contenido en un plan que implica ordenar una serie de actividades y recursos en el tiempo, con la intención de resolver problemas y generar beneficios para la comunidad.

La formulación de un proyecto debe realizarse de manera participativa, tomando en cuenta el punto de vista de todas las personas potencialmente beneficiarias del mismo. 
4. Actividad física: en este estudio se recurrió a dos corrientes epistemológicas y conceptuales de la actividad física: las establecidas por Pedraz y la CDC (ver Tabla 1) (Calderón y Alzate, 2010), que permiten reflexionar acerca de los propósitos que se definen para esta y facilita un acercamiento a la estructuración de nuestro propio concepto.

\section{Metodología}

En este proyecto se retoman aspectos metodológicos propios de la investigación cualitativa y cuantitativa para la recolección y triangulación de datos, caracterización y comparación de los mismos. En correspondencia con los objetivos planteados, se describen a continuación los enfoques e instrumentos utilizados y desarrollados en esta investigación:

- Análisis documental y comparativo de : a) marco legislativo, político y administrativo, los programas exitosos de actividad física en la Guajira, Bogotá y Antioquia, y b) matrices de los programas y del video-audio sobre el programa de movilidad ciudadana, presentado por un funcionario del gobierno local de Bogotá.

- Entrevistas semiestructuradas dirigidas a los responsables de los programas de actividad física, que tuvieron como objetivo identificar y caracterizar las concepciones pedagógicas y didácticas de las políticas, planes y programas de actividad física.

- Cuestionario. Instrumento traducido y adaptado del National Fitness Institute, por el GPAF, que fue denominado "Salud y actividad física". Presenta categorías relacionadas con la condición física, la pedagogía y didáctica y el conocimiento de las políticas por parte de los beneficiarios. De acuerdo a las necesidades de esta investigación, se estructuraron dos cuestionarios, uno de preguntas cerradas, con escala Likert; y otro de preguntas abiertas de tipo cualitativo.

La población objeto de estudio fueron profesionales que orientan los programas "Guajira viva y activa", "Por su salud muévase pues" y "Muévete Bogotá", pertenecientes a los departamentos de Guajira, Antioquia y Bogotá. Se aplicó el cuestionario "Salud y actividad física" a un total de 158 personas, participantes en programas oficiales de actividad física, 51 en Bogotá, 55 en Antioquia y 52 en Guajira.

\section{Resultados y análisis}

En la aplicación del instrumento "Actividad física y salud" se obtuvo información que se organizó y analizó en tres categorías: a). políticas, planes, programas y proyectos, b) pedagogía y didáctica, c). actividad física.

\section{a) Políticas, planes, programas y proyectos}

En la revisión documental de los Manifiestos de actividad física de las organizaciones del sector deportivorecreativo nacional e internacional, se evidenció:

- Carencia de un componente educativo y pedagógico en las políticas, planes programas y proyectos de actividad física.

- Suficiente normatividad para orientar la práctica de actividades deportivas, recreativas y de actividad física, para lo cual el Estado debe garantizar el control y evaluación permanente de estas.

- Existencia de procedimientos adecuados para la construcción y aplicación de políticas deportivas y recreativas en el sector.

- Desconocimiento de las políticas relacionadas con la supresión de competencias que Coldeportes mantenía con la educación física, cuya responsabilidad se trasladó al Ministerio de Educación Nacional.

El análisis de las políticas en educación permite ver dos tendencias significativas en la forma de comprender la actividad física: una, la manera funcionalista desde la cual ha sido utilizado el discurso de la educación, y la segunda, la educación física al servicio de los intereses del Estado.

La primera tendencia muestra la evolución histórica de la educación física en la legislación colombiana desde épocas de la Colonia. Estas fueron:

- Educación física con tendencia militar (1826).

- Desarrollo intelectual, moral y físico (1826).

- Calistenia y corrección postural (1903).

- Enfoque higiénico-médico (1925).

- Restauración fisiológica y preparación de nuevas fuerzas de trabajo (1936). 
Concepciones pedagógicas y didácticas de las políticas, planes, programas y proyectos de actividad física a nivel local y nacional. Alberto Calderón García, Olegario de Jesús Puentes Torres,

Víctor Hugo Durán Camelo, Ramiro Andrés Alzate Lubo y Gloria Viviana Barinas Prieto

- Deportivista (1968).

- Globalización y diversidad con enfoque laboral (1995).

La segunda tendencia encontró que las políticas actuales en relación con la educación física y la actividad física, tienen una influencia muy marcada del sistema económico imperante, que por medio de metáforas intenta formar un ser humano "físicamente bien educado" saludable y productivo; desde esta perspectiva, las políticas intentan garantizar que los ciudadanos mantengan niveles adecuados de salud que les permitan continuar con sus deberes laborales, surgiendo términos como preservación, competencias, desarrollo sostenible.

Respecto a los términos educativos encontrados, se observa que están acompañados de otros conceptos que desplazan el proceso de formación y lo ubican en el escenario laboral como formación y educación para el trabajo.

No hay un amplio estudio sobre el conocimiento de las políticas, lo cual permite plantear la necesidad de trabajar sobre la gestión por parte de las áreas responsables.

A través del cuestionario "Salud y actividad física", se tuvo presente el análisis de las siguientes variables:

1. Conocimiento de políticas a nivel local, distrital o nacional en actividad física.

2. Conocimiento de planes de actividad física.

3. Conocimiento de algún programa o proyecto en actividad física desarrollado por el instituto municipal o distrital de deportes y recreación.

4. Desarrollo de algún proyecto de actividad física o ejercicio físico en la localidad o en el barrio en que se reside.

5. Implementación de programas o proyectos de actividad física en su sitio de trabajo.

Las cifras relacionadas con las opiniones de los participantes de estos programas confirman los apartados anteriores, no siendo muy alentadoras por cuanto:

- El 44\% conoce medianamente las políticas que regulan la actividad física a nivel local, distrital o nacional.

- $\quad 42 \%$ y $44 \%$ conocen los planes de actividad física y sus programas o proyectos, respectivamente, ejecutados por el respectivo instituto municipal o distrital de deportes y recreación.
- $\quad$ El 41\% da cuenta de la existencia de programas de actividad física en su localidad o en el barrio que reside.

- El 40\% expuso que en su sitio de trabajo no se implementan programas o proyectos de actividad física, lo cual significa que las empresas hacen caso omiso con lo manifestado en la legislación (ver Figura 1).

Figura 1. Opiniones de los usuarios con relación a los programas, planes y proyectos

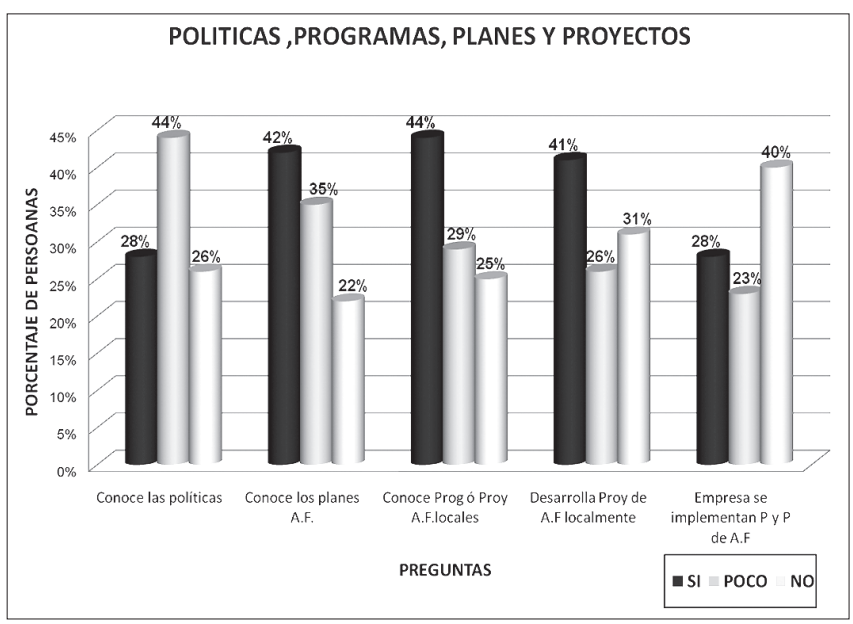

La acción de las políticas públicas debe atender un espectro mayor de circunstancias, ámbitos y relaciones que tienen que ver con la calidad de vida y con posibilitar la salud del ciudadano; esto implica una complementariedad, una relación dialógica y dialéctica entre el ciudadano y el Estado que formula las políticas públicas.

Esta tendencia hace necesario potenciar sujetos "activos" y propositivos, que conformen una estructura de relación horizontal frente al Estado, requiere de procesos que impliquen una intención educativa y/o pedagógica con relación a su propósito, es decir, "potenciar al ciudadano integralmente saludable".

\section{b) Pedagogía y didáctica}

Se utilizó como metodología el análisis documental, la entrevista estructurada y el análisis sobre los elementos comunicativos, obteniendo los siguientes resultados:

- En Colombia no se podría generalizar sobre una única y exclusiva forma de asumir la pedagogía y la didáctica presente en los espacios de actividad física.

- La promoción, difusión y comunicación de la actividad física es más espontánea que planificada. 
- El desarrollo académico de la actividad física es moderado debido a la metodología utilizada, resultado de la preparación individual de los instructores.

- Algunos de los instructores son profesionales en educación física o deporte pero no tienen experiencia en procesos de investigación, ni formación profesional específica en actividad física.

- Los conceptos pedagogía y didáctica tienen correspondencia con términos que, en la mayoría de los casos, dan a entender que en los planes de actividad física existe una tendencia más ligada a la teoría comunicativa que a la educativa como tal.

- La interpretación de los modelos pedagógicos deja ver que la retroalimentación es asumida de distintas formas, validando la postura o intención pedagógica que la sustenta.

A través del cuestionario "Salud y actividad física", se presentaron a los usurarios variables que estaban relacionadas con aspectos como:

1. La diversión y motivación durante la sesión de actividad física.

2. Atención del desempeño por parte del instructor.

3. Planeación, organización y ejecución de la sesión.

4. Utilización de ayudas y recursos en la sesión.

5. Esfuerzo e impacto logrado durante la sesión.
Acorde con este marco, se encontró:

- El 50\% respondió estar muy satisfecho con la planeación y organización de la sesión, la metodología y la seguridad, el cuidado, la motivación, la atención y explicación, lo cual quiere decir que es buena la interacción pedagógica y didáctica entre usuario e instructor.

- Por otra parte, menos de la mitad disminuyó su satisfacción por la complejidad de los ejercicios, el esfuerzo al que fueron sometidos y el grado de diversión de la sesión (ver Figura 2).

Desde la satisfacción de los practicantes se encontró:

a) Un valor formativo para las sesiones de actividad física.

b) Pocos estiman su programa de actividad física como un entrenamiento o como ejercicio de alto impacto.

c) En este tipo de prácticas predomina el estilo "comando directo", según el espectro de Mosston y Ashworth (1994), que es la estrategia más relevante en las actividades relacionadas con la actividad física.

En conversación sostenida con los usuarios, se dedujo que ellos tienden hacia el asistencialismo, es decir, a recibir los servicios de actividad física y salud desde las diferentes instancias estatales, más no a participar o integrarse directamente con las dinámicas de construcción de políticas públicas y de autogestión de planes y programas.

Figura 2. Aspectos pedagógicos y didácticos

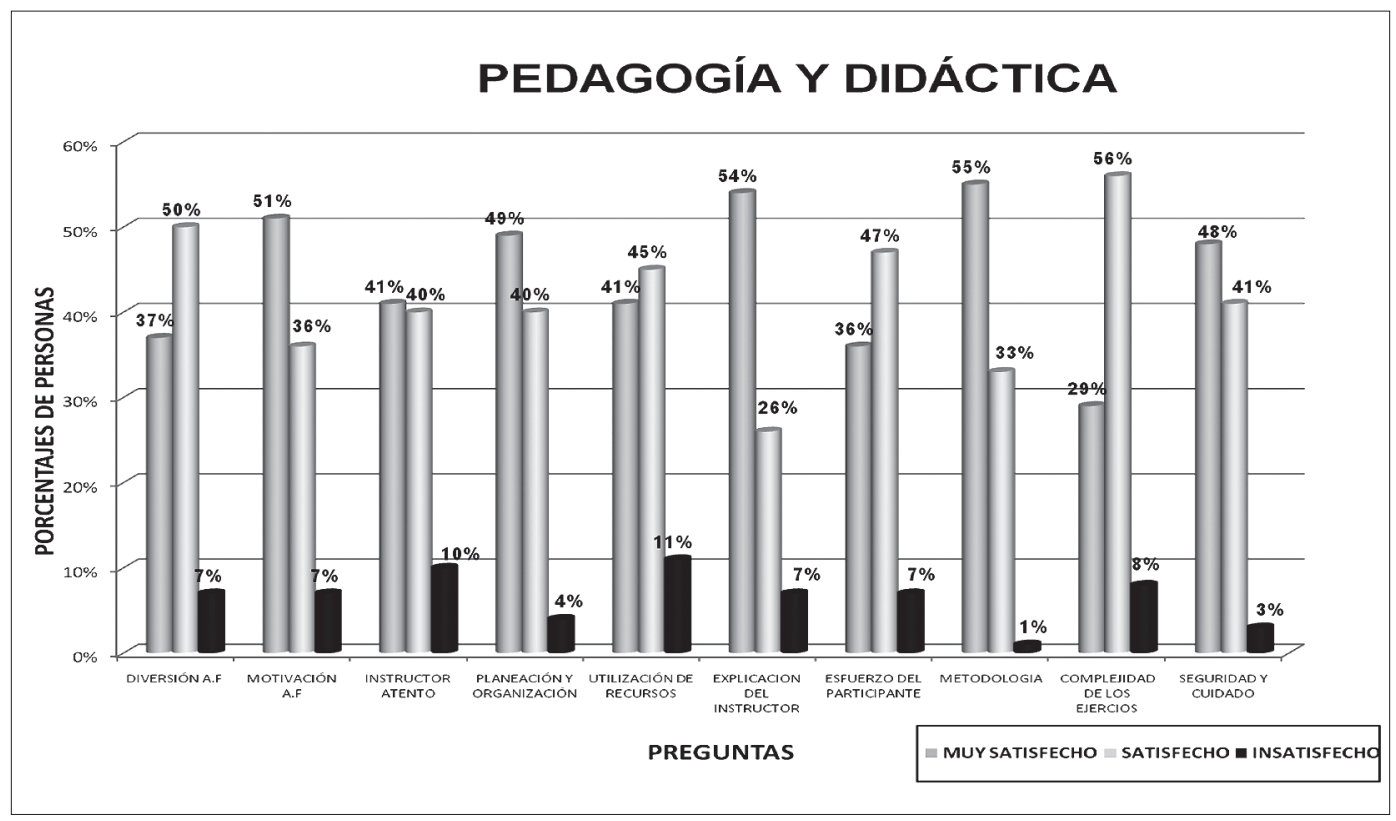


Concepciones pedagógicas y didácticas de las políticas, planes, programas y proyectos de acti-

vidad física a nivel local y nacional. Alberto Calderón García, Olegario de Jesús Puentes Torres,

Víctor Hugo Durán Camelo, Ramiro Andrés Alzate Lubo y Gloria Viviana Barinas Prieto

La pregunta que se refiere a las concepciones pedagógicas y didácticas, lleva implícito un cuestionamiento acerca de los sujetos (directivos) que las formulan y el fundamento epistémico, lo cual incide directamente en el conjunto de acciones de las mismas y la manera en que son percibidas por el usuario final.

Las concepciones permiten dar cuenta de la estructuración teórica de los directivos, su análisis facilita interpretar y evidenciar cual es la tendencia pedagógica-didáctica que pretenden orientar. Otro factor es la correspondencia entre la concepción pedagógica-didáctica y la intención educativa de los programas de actividad física; estos deben afectar en lo posible a la mayor cantidad de personas (usuarios), por medio de diferentes estrategias en las que se supondría puede encontrarse explícito un fundamento, tanto pedagógico como didáctico, en todas las fases del proceso.

Respecto a los actores existen también distinciones, entre los cuales se reconocen los usuarios, los promotores y los gestores de las políticas, planes, programas y proyectos. Los primeros son tenidos en cuenta como sujetos pasivos del proceso (receptores de información); los segundos son objeto de cualificación en tanto se asumen exclusivamente como ejecutores del programa; y los terceros son portadores del único saber válido y transmisible (emisor). Lo anterior es observable cuando conceptos como promoción, difusión, información y formación se plantean como necesidad de las políticas, planes, programas y proyectos, formulando los objetivos o metas propuestas de manera unidireccional.

La legislación estudiada para el marco legal muestra el desarrollo de los programas, referenciando distintas leyes, artículos, decretos, acuerdos, desde un enfoque vinculado a contextos como la salud ocupacional, salud pública, el tiempo libre y la práctica deportiva, siendo escasa la alusión a legislaciones de vital importancia, como es el caso de la Ley 115/94 y la 1064/06, que sustentan las necesidades educativas a nivel formal y de apoyo y el fortalecimiento de la educación para el trabajo y el desarrollo humano.
En resumen, el discurso pedagógico-didáctico se reemplaza en estas propuestas, circulando otro discurso sustentado en la teoría comunicativa. En el caso de la didáctica la situación es similar, puesto que es entendida de manera instrumental, asumiendo que las únicas estrategias efectivas que pueden ser utilizadas en las fases de implementación son necesariamente los medios impresos y audiovisuales como cartillas, afiches, volantes, camisetas y videos.

Lo anterior toma importancia en la medida en que las fases de promoción e implementación se asuman desde el enfoque comunicativo pedagógico; esto implica reconocer a los sujetos presentes, que la información circule en doble vía, que se haga uso de la retroalimentación con el fin de generar una acción crítica de la realidad, generar aprendizajes y reconstruir la propuesta planteada a partir de la experiencia, vivencia e interacción de los sujetos presentes.

\section{c) Actividad física}

Analizando los resultados obtenidos con la técnica descriptiva, se estableció (ver Figura 3):

- $\quad$ El 38\% hace actividad física por lo menos cinco veces por semana, lo cual está de acuerdo con las recomendaciones de las principales entidades científicas encargadas del estudio de la actividad física a nivel mundial.

- El 51\% realiza la mayoría de las veces una adecuación y proceso de adaptación en su práctica de actividad física, a través del calentamiento previo y el estiramiento posterior.

- El $42 \%$ refiere que rara vez desarrolla hábitos adecuados para la práctica, relacionados con el concepto científico de monitoría de la intensidad de la frecuencia cardiaca de acuerdo al tipo de actividad, sus objetivos y riesgos.

- El 66\% disfruta el tipo de actividad que realiza.

- El 47\% respondió que la mayoría de las veces sí tienen en cuenta la resistencia cardiovascular y muscular, fuerza y flexibilidad en la sesiones. 
Figura 3. Actividad física usuarios

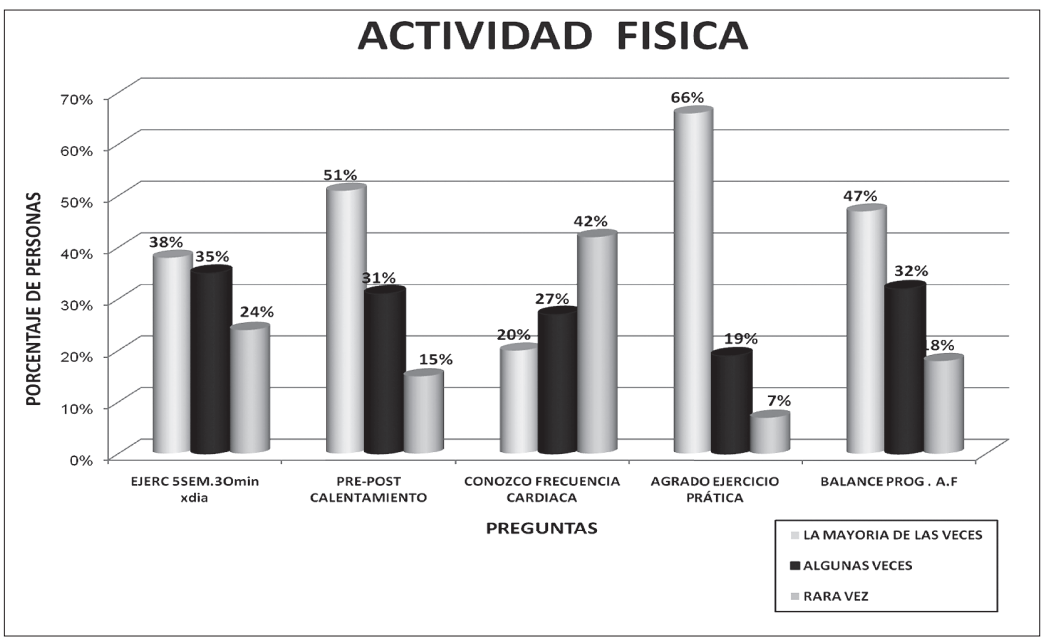

En conversaciones sostenidas con los practicantes de los programas escogidos por Coldeportes como los más exitosos, se deduce que la intención educativa de estos programas está centrada en informar los efectos negativos del sedentarismo, promover el cambio de hábitos en la población y masificar la participación en las actividades programadas.

Los procesos pedagógicos de los programas de actividad física en Colombia, demuestran resultados positivos en la creación de hábitos en los usuarios, quienes consideran que conocen y han percibido los beneficios de la actividad física en su vida cotidiana, circunstancia que se refuerza con el componente didáctico de las sesiones.

La falta de un énfasis en el componente educativo y pedagógico, limita a los usuarios en el conocimiento de los beneficios relacionados con la salud física, emocional y mental y su estrecha relación con la regularidad e intensidad de la práctica. Se hace indispensable que el usuario identifique lo que está haciendo y para qué lo está haciendo, evitando así caer en la monotonía, la desmotivación y en la deserción de los programas.

En el panorama actual se identifican dos posturas diferentes frente al concepto de salud: una de ellas es la forma de ver la salud como "ausencia de enfermedad", lo que demandaría del Estado y las políticas de salud pública una actitud asistencialista en pro de contrarrestar la enfermedad en los ciudadanos, debido a que se estaría frente un sujeto "pasivo" receptor de las acciones estatales, que buscan alejar la enfermedad del individuo.
La segunda tendencia de la salud hace una mirada más compleja, dependiente de la interacción adecuada entre las múltiples dimensiones humanas; es decir, la salud depende del bienestar físico, psíquico, laboral, afectivo y social en su conjunto.

La salud en sí misma cumple funciones sociales, políticas y económicas, con diferencias marcadas por la tendencia o "concepción de salud" desde la cual se gestione. Es claro cómo existe una relación directa entre las políticas públicas relacionadas con la salud y el campo laboral: el "ciudadano no enfermo" o "el ciudadano integralmente saludable", de quien se requiere su integración a las tendencias de eficacia, eficiencia y de productividad laboral.

Esta falencia ha tenido como resultado que para dar solución a las críticas condiciones de accesibilidad a derechos fundamentales como el mantenimiento de un buen estado de salud, prime la atención de la enfermedad, cuando también es posible lograr el buen estado físico a través de programas que contribuyan a la prevención de nuevas patologías, a través de la participación en programas de actividad física.

Surge la necesidad de incluir estilos de vida saludables en los habitantes del país, para que sea relevante la práctica de actividad física de forma regular y esta complemente las actividades diarias de los habitantes.

En algunos municipios del país se han intentado establecer sin éxito programas de actividad física para sus habitantes, debido a que, generalmente, se reproducen modelos que han sido propuestos para poblaciones en 
Concepciones pedagógicas y didácticas de las políticas, planes, programas y proyectos de actividad física a nivel local y nacional. Alberto Calderón García, Olegario de Jesús Puentes Torres,

Víctor Hugo Durán Camelo, Ramiro Andrés Alzate Lubo y Gloria Viviana Barinas Prieto

contextos diferentes. Es necesario establecer diagnósticos previos a cada uno de estos programas que permitan identificar las características de las poblaciones, y así construir programas de actividad física que realmente respondan a sus necesidades y características e incidan positivamente en el estado de salud de la población.

Es importante resaltar la trascendencia que comienzan a tener los programas de actividad física en el contexto latinoamericano y global, convirtiéndose en motivo de interés en las agendas gubernamentales tanto a nivel mundial, como nacional y local.

No obstante, pese a los múltiples esfuerzos de los gobiernos y administraciones, persisten problemas en la respuesta dada a la convocatoria de los habitantes a este tipo de iniciativas. El resultado es la atomización de propuestas cortoplacistas, inmediatistas y con un carácter que busca solucionar la problemática bajo un modelo asistencial. Esto genera proyectos distantes de las comunidades, pues no responden a sus intereses, necesidades y expectativas, y que finalmente no los convierte en protagonistas de las soluciones.

\section{Conclusiones}

Conocimiento implícito en la actividad física. Se hace evidente la necesidad de que los directivos que formulan las políticas, planes, programas y proyectos, en contraste con los programas "Exitosos de Actividad Física a nivel regional y nacional, ", redimensionen y resignifiquen la actividad física, no como un conjunto de acciones asistencialistas, sino como un saber pedagógico que requiere de una estructura educativa que posibilite procesos de enseñanza-aprendizaje en la población, basados en un modelo de relación multidireccional, horizontal, dialógico, autónomo y crítico. Esto implica la acción de equipos interdisciplinares que sean liderados por profesionales de la actividad física, educación física, deporte, cultura física y recreación, que garanticen nuevas comprensiones de la actividad física como fenómeno educativo y sociocultural.

Educación y formación en el ámbito de las políticas, planes, programas y proyectos de actividad física. Desde la perspectiva de la educación y la formación, Carlos Vasco ha afirmado que estos conceptos están claramente definidos como procesos de construcción del ser humano. Sin embargo, los referentes revisados invisibilizan la importancia del proceso educativo e ins- trumentalizan la formación, haciéndola equiparable a la cualificación referida a "contenidos instrumentales" de las personas responsables.

Los medios masivos y formación. Reiterar ante los directivos la circulación de la información a través de los medios de comunicación masiva o la utilización de algunas estrategias didácticas, no compensa ni reemplaza los aprendizajes que se pueden estructurar en la población a partir de un adecuado proceso educativo.

Desarticulación. Se observó poca coherencia y cohesión entre las personas y grupos que se encargan de la gestión de las políticas, y quienes son responsables de la ejecución y evaluación. También el desconocimiento de las políticas y planes por parte de los usuarios de los programas, lo cual repercute en la poca adherencia y sentido de pertenencia con estos, incidiendo en la generación de sobrecostos por la repetición de programas por parte de diferentes entidades públicas y privadas que buscan un mismo objetivo.

Generación de hábitos en los usuarios de los programas de actividad física. Se trata de mostrar la falta de un componente educativo y pedagógico fuerte y estructurado por parte de los programas en actividad física, de forma que estos puedan generar procesos en los usuarios para la adquisición de hábitos adecuados y consecuentes con la práctica realizada.

Participación e inclusión. Ha habido muy poca participación y vinculación de los usuarios en los programas de actividad física. Incluso, se percibe un gran desconocimiento de las convocatorias de programas de carácter oficial y menos participación aún en la construcción de las políticas públicas.

Se encontró que en la actualidad las políticas, planes, programas y proyectos se construyen y desarrollan desde una perspectiva administrativa que margina en parte las intenciones educativas, las estrategias pedagógicas y didácticas, y que finalmente poco repercuten en la implementación de las primeras.

Actividad física y política social. Se da poca importancia a la política social como una forma de intervención del Estado en la sociedad civil, para gestar servicios y brindar programas de deporte, salud, seguridad, vivienda, educación y ocio, y en este caso para fomentar la actividad física y lograr un cambio en el orden social. 
Ser pioneros. El grupo de investigación, luego de su labor de consulta, resalta su labor pionera en la aproximación conceptual, metodológica y operativa de las teorías y prácticas administrativas con aquellas de la pedagogía, didáctica y disciplinares, como es el campo de la actividad física.

\section{Recomendaciones}

- Propiciar por parte del gobierno nuevas movilizaciones sociales en torno a la construcción de políticas públicas y planes de desarrollo, lo cual permitiría evaluaciones institucionales y generaría posibilidades de cambios significativos de orden social.

- Explorar, en estudios de corte educativo, la aplicación pedagógica y didáctica de la actividad física, para resaltar la importancia del componente.

- Continuar analizando diversas variables para proponer nuevos campos investigativos en torno a la producción y construcción de conocimiento pedagógico, que sirvan como base para la formación de profesionales en el campo de la administración deportiva, la pedagogía y otros propios de la actividad física y el deporte.

- Mejorar las estrategias de información y comunicación de los fundamentos teórico-prácticos que conciernen a lo político, pedagógico, disciplinar y la educación del usuario.

- Establecer una relación dialógica efectiva entre los procesos pedagógicos y los procesos comunicativos.

- Plantear a la comunidad académica que en el camino hacia la elaboración de un constructo teórico de la actividad física, se debe tener presente la necesidad de unificar los términos, no solo desde el punto de vista epistemológico sino también desde la praxis, para evitar yuxtaposiciones, polisemia y confusión.

- Reconocer el valor de lo pedagógico y didáctico como herramientas fundamentales para lograr el desarrollo humano.

- Motivar a las instituciones promotoras de la actividad física a trabajar en un marco interinstitucional, intersectorial e interdisciplinario que sea una realidad en la construcción del Sistema Nacional del Deporte.

\section{Referencias}

Calderón, A. y Alzate, R. (2010). Conocimiento profesional de los profesores de Educación Física colombianos y mexicanos, sobre Actividad Física. Revista Lúdica Pedagógica 2(15), 103-115.

Camacho, H. (2003). Pedagogía y didáctica de la Educación Física. Armenia: Ed. Kinesis.

Flórez R., F. (1994). Hacia una pedagogía del conocimiento. Bogotá: McGraw-Hill.

Gallego, R. y Pérez, R. (1988). Concepciones epistemológicas, pedagógicas y didácticas. Bogotá: UPN.

Lucio, R. (1996). La construcción del hacer y del saber hacer. En Pedagogía y educación popular. Revista Aportes (41). Bogotá: Dimensión Educativa.

Martínez, C., Guzmán, G. y Calderón, A. (2005). Educación, pedagogía y didáctica en la escuela: encuentros y desencuentros. Revista Lúdica Pedagógica 2(10), 113120.

MEN. (1994). Ley 115 de febrero de 1994.

MEN. (2006). Ley 1064 de julio 26 de 2006.

Moscovici, S. (1979). El psicoanálisis, su imagen y su público. Buenos Aires: Editorial Huemul.

Mosston, M. \& Ashworth, S. (1994). Teaching physical education. (4th ed.). New York: Macmillan.

Pedraz, M. (1987). Teoría pedagógica de la actividad física. Madrid: Ed. Gymnos S.A.

Roth, A. (2006). Discurso sin compromiso. La política pública de derechos humanos en Colombia. Bogotá: Ediciones Aurora.

Sistema Nacional del Deporte. (1995). Ley 181 de enero de 1995.

Vasco, C. (1985). Algunas reflexiones sobre la Pedagogía y la Didáctica. En: Pedagogía, Discurso y Poder. Santa Fe de Bogotá: CORPRODIC.

Zuluaga, O. et al. (1988). Pedagogía, didáctica y enseñanza. Educación y Cultura (14). Bogotá. 